\title{
2010 CAEP/ACMU Scientific Abstracts Keyword Index
}

KEYWORD

ABSTRACT No.

A

abdominal pain

159

accreditation

165

acetaminophen poisoning

acute coronary syndromes

66,79

44

79

149

acute myocardial infarction

administrative database research 24,78 ,

91,161

administrative database study $23,80,116$

admission delays

27

admission predictors

22,83

adolescents

adrenal function

adverse drug events

adverse events

agonal breathing

AGREE

agreement

airway management

airway management courses

allergy testing

alternative funding arrangement $\quad 74$

ambulatory care

anaphylaxis

anticoagulation

antiemetic therapy

asthma

atrial fibrillation

$12,22,25,28,46$, $47,56,88,129$

automated external defibrillators

$53,99,127$

56,147

68

73

14,105

$19,36,45,55$,

15
143

71

63

101

127

74

35

68

B

barrier analysis $\quad 136$

best practices $\quad 70$

bicycling behaviour $\quad 125$

bleeding events 147

blended practice $\quad 148$

blunt thoracic trauma 42

blunt trauma 92

brain natriuretic peptide $\quad 115$

breaking bad news $\quad 113$

bronchiolitis 146

C

Canada-US comparisons

CanMEDS

$13,94,98$ cardiac arrest

cardiac biomarkers

cardiac monitoring

cardiopulmonary resuscitation $\quad 9,15$

cardioversion

CARMS

case-control study

case presentations

CDU implementation

CEDIS

central venous catheter insertion

CHADS score

chest pain

chest tube insertion

$54,93,119,124$

chief complaint classification

child abuse

clinical competence

clinical criteria

clinical decision rule

clinical decision units

$5,11,92,105,136$

clinical education guideline

clinical efficiency

clinical practice guidelines

clinical prediction rule

clinical productivity

codeine

community-acquired pneumonia

community emergency medicine

community physicians

comparative effectiveness

consensus methodology

consultation delays

consultation patterns

continuing medical education

controlled trial

COPD

CPR training

credentialing

critically appraised topic

CT chest

CT utilization

CTAS

cycling injuries

$71,83,111,144$

D

delayed adverse outcomes

Delphi methodology

descriptive analysis
95,141

111,144

$3,18,75$

89

136

12

110

106

164

141

34

70

56

160

144

118

13,67

99

143

57

20, 106

$42,44,134$

37,38

26

154

150

142

61

94

80

108

123,150

81

61,121

58

155

163

42

23, 91

134

13,16

153,154

162 didactic education

123

disaster medicine 52, 114

discrepant interpretations

126

disposition decision

55

distance learning $\quad 164$

Doppler ultrasonography $\quad 138$

drug related visits

14

dysarthria

49

E

ED design

EDIS

31

ED length of stay $\quad 116$

ED misuse $\quad 158$

ED throughput

education research

educational research

electrical cardioversion

$52,107,112$

63,103

60, 88

electrocardiography

130

electrode pad positioning

60

electronic decision support

20

EM training streams

139

emergency crowding $4,8,17,24,27,32$, $41,57,64,65,80,82,86$,

$88,95,121,122,124,128,149$

emergency medical services

165

emergency medicine primer

90

emergency radiography

126

emergency ultrasound

$1,7,155$

etomidate

2,77

F

family medicine stream $\quad 148$

febrile neutropenia 20

fee for service $\quad 74$

femoral fractures $\quad 72$

femoral nerve block $\quad 72$

fomepizole $\quad 135$

fractile response $\quad 74$

free text entries 34

full capacity protocols $\quad 65$

functional decline 33

G

gap analysis

73

gastroenteritis

39,68

geriatrics 33,137, 145, 158

Glasgow Outcome Scale

59

GRADE

143 
graduate medical education guideline compliance

H

$\mathrm{H} 1 \mathrm{~N} 1$

$10,50,62,76,109,161$

$\mathrm{HCV}$

head trauma

health records review $22,46,97,99,100$

heart failure

helmet legislation

high fidelity simulation

HIV

hydronephrosis

hypertonic saline

hyperventilation

hypoglycemia

\section{I}

imaging strategies

impact analysis

implementation research

independent elders

infection control

influenza assessment clinics

injection drug use

injection drug users

injury circumstances

injury patterns

internal validation

international EM

interprofessional learning

interrater agreement

intra-articular lidocaine

intracranial bleeding

ischemic stroke

\section{K}

ketamine propofol

knowledge transfer

knowledge translation

120

9

146

L

Lean management

learner self-assessment

liver injury

long-term outcomes

low acuity ED patients

low acuity patients

LWBS

M

mean arterial pressure

medical direction

medical evacuation

medico-legal considerations

mild traumatic brain injury

minor head trauma

minor trauma
110

73

118

115

125

52,112

132

motor vehicle accidents

MRSA

multisource feedback

$\mathbf{N}$

narcotics

needs assessment

night shifts

nonspecific vertigo

nonurgent visits

\section{O}

observation units

online survey research

operations research

order sets

OSCE

$\mathbf{P}$

pain management

pandemic influenza

pandemic planning

panic disorder

paramedic decision-making

paramedic training

patient advocacy

patient flow

patient safety

patient satisfaction

$14,16,70,93,126$

152

113

111

48

84

49

pediatric emergency medicine

$72,104,108,160$

pediatric mental health

pediatric pain management

pediatric procedures

pediatric resuscitation

performance standards

physician acceptability

physician efficiency

physician productivity

physician workload

pilot studies

plastic surgery

pneumonia severity index

pneumothorax

podcasts

population research

practice variability

practice variation $46,68,91,95,96,146$

prehospital

prehospital analgesia

prehospital dispatch prehospital transport

pre-post study design

pre-post study designs

pretriage

prevalence

PRIDE scoring system

procedural confidence
133

87

94

153,163

150

85

78

122

53,64

21

8

53

30

26

76, 109, 161

$54,119,130$

$63,67,101$

156

31

104

160
86

96

142

112

165

105

155

90

90

120

108

100

1

123

$25,66,129$

28,47

3,21

166

75

158

101

103

50

54

115

142 procedural sedation

$19,36,45,77$,

120,140

prognostic study

prospective study

12

19

provincial database

25

public health surveillance $\quad 144$

pulmonary edema $\quad 163$

pulmonary embolism

162

Q

qualitative research

$15,18,141$

quality of care

\section{$\mathbf{R}$}

Radiation Casualty Assessment Tool 114 randomized controlled trial 26, 48

rapid assessment zones $\quad 128$

real-time survey $\quad 55$

recidivism 78

reliability $\quad 34,111$

renal colic 7

research methodology $\quad 17$

research methods 40

residency program selection $\quad 139$

residency selection $\quad 110$

residency training

$51,69,81$,

$102,107,117,152$

residents as teachers 69

resuscitation 30

resuscitation skills $\quad 102$

retrospective review $\quad 157$

revisit rates 62

Rh immune prophylaxis $\quad 97$

risk prediction $\quad 79$

risk stratification $\quad 5,11$

Romanian EM

152

rural emergency medicine 154,156 ,

157,162

S

screening aids 119

self-reported confidence $\quad 67$

sepsis 106

seroprevalence study $\quad 132$

service cuts 156

shiftwork 85

shoulder dislocation $\quad 48$

simulation training $\quad 51,98,102$,

$113,117,160$

simulation

30

specialized care models $\quad 86$

specialty follow-up $\quad 127$

stethoscope contamination $\quad 87$

stroke prevention $\quad 43$

study screening $\quad 40$

survey research $9,28,47,58,69$,

$85,96,98,122,139,148$

survey study

51,117 
symptom description

syncope

134

syndromic surveillance

systemic review

systematic review

$50,76,109$

4

$1,2,6,17,32$ $41,44,60,64,65$, $89,118,128,135$

system-wide interventions

32,100 ,

$121,124,149$

T

tabletop exercises

task times

31

37,38

3

97

termination of resuscitation

threatened miscarriage throughput analysis

throughput measurements

time-motion analysis

time-motion study

toxic alcohols

trainee impact

transient ischemic attack

traumatic brain injury

triage

triage liaison physician

triage nurse order

troponin
$\mathbf{U}$

ultrasound training unplanned revisits

urethral visibility

urgent outpatient evaluation

urinary tract infection

V

venous thromboembolism

ventilation perfusion scanning

videoconferencing

W

waiting room care

waiting room medicine

warfarin

wellness curriculum
137

29

43

29

138

138

164

8

93

84,147

81 